\title{
Unilateral Herpes Zoster Ophthalmicus Results in Bilateral Corneal Nerve Alteration
}

\section{Citation}

Hamrah, Pedram, Andrea Cruzat, Mohammad H. Dastjerdi, Harald Prüss, Lixin Zheng, Bashar M. Shahatit, Hasan A. Bayhan, Reza Dana, and Deborah Pavan-Langston. 2013. "Unilateral Herpes Zoster Ophthalmicus Results in Bilateral Corneal Nerve Alteration." Ophthalmology 120 (1) (January): 40-47. doi:10.1016/j.ophtha.2012.07.036.

\section{Published Version}

10.1016/j.ophtha.2012.07.036

\section{Permanent link}

http://nrs.harvard.edu/urn-3:HUL.InstRepos:34814012

\section{Terms of Use}

This article was downloaded from Harvard University's DASH repository, and is made available under the terms and conditions applicable to Other Posted Material, as set forth at http:// nrs.harvard.edu/urn-3:HUL.InstRepos:dash.current.terms-of-use\#LAA

\section{Share Your Story}

The Harvard community has made this article openly available.

Please share how this access benefits you. Submit a story.

\section{Accessibility}


Ophthalmology. 2013 January ; 120(1): 40-47. doi:10.1016/j.ophtha.2012.07.036.

\title{
Unilateral Herpes Zoster Ophthalmicus Results in Bilateral Corneal Nerve Alteration:
}

\author{
An In Vivo Confocal Microscopy Study
}

Pedram Hamrah, MD1 , Andrea Cruzat, MD¹, Mohammad H. Dastjerdi, MD¹, Harald Prüss, MD², Lixin Zheng, MD ${ }^{1}$, Bashar M. Shahatit, MD ${ }^{1}$, Hasan A. Bayhan, MD ${ }^{1}$, Reza Dana, MD, $\mathbf{M P H}^{1}$, and Deborah Pavan-Langston, MD ${ }^{1}$

${ }^{1}$ Ocular Surface Imaging Center and Cornea \& Refractive Surgery Service, Massachusetts Eye \& Ear Infirmary, Department of Ophthalmology, Harvard Medical School, Boston, Massachusetts

${ }^{2}$ Department of Pathology, Harvard Medical School, Boston, Massachusetts

\begin{abstract}
Purpose-Herpes zoster ophthalmicus (HZO), thought to be a unilateral disease, results in loss of corneal sensation, leading to neurotrophic keratopathy. This study aimed to analyze bilateral corneal nerve changes in patients with HZO by in vivo confocal microscopy (IVCM) and their correlation with corneal sensation as a measure of nerve function.
\end{abstract}

Design-Prospective, cross-sectional, controlled, single-center study.

Participants-Twenty-seven eyes with the diagnosis of HZO and their contralateral clinically unaffected eyes were studied and compared with normal controls $(\mathrm{n}=15)$.

Methods-In vivo confocal microscopy (Confoscan 4; Nidek Technologies, Gamagori, Japan) and corneal esthesiometry (Cochet-Bonnet; Luneau Ophthalmologie, Chartres, France) of the central cornea were performed bilaterally in all patients and controls. Patients were grouped into normal $(>5.5 \mathrm{~cm})$, mild $(>2.5-5.5 \mathrm{~cm})$, and severe $(<2.5 \mathrm{~cm})$ loss of sensation.

Main Outcome Measures-Changes in corneal nerve density, total nerve number, main nerve trunks, branching, and tortuosity were evaluated after IVCM and were correlated to corneal sensation, disease duration, and number of recurrences.

Results-Eyes with herpes zoster ophthalmicus had a significant $(P<0.001)$ decrease in total nerve length $(595.8 \pm 358.1$ vs. $2258.4 \pm 989.0 \mu \mathrm{m} /$ frame $)$, total number of nerves $(5.4 \pm 2.8$ vs. $13.1 \pm 3.8)$, number of main nerve trunks ( $2.3 \pm 1.1$ vs. $4.7 \pm 1.2)$, and number of nerve branches (3.2 \pm 2.3 vs. $8.4 \pm 3.7)$ as compared with controls. In the contralateral clinically unaffected eyes, total nerve length $(1053.1 \pm 441.4 \mu \mathrm{m} /$ frame), total number of nerves $(8.3 \pm 2.9)$, and main nerve trunks $(3.1 \pm 1.0)$ also were decreased significantly as compared with controls $(P<0.01)$. Reduced nerve density, total nerve count, main trunks, and tortuosity was correlated significantly with corneal sensation across all subgroups $(P<0.001)$.

Conclusions-Patients with unilateral HZO demonstrated a profound and significant bilateral loss of the corneal nerve plexus as compared with controls, demonstrating bilateral changes in a

(C) 2013 by the American Academy of Ophthalmology.

Correspondence: Pedram Hamrah, MD, Cornea Service, Massachusetts Eye \& Ear Infirmary, Harvard Medical School, 243 Charles Street, Boston, MA 02114. pedram_hamrah@meei.harvard.edu.

Financial Disclosure(s):

The author(s) have no proprietary or commercial interest in any materials discussed in this article. 
clinically unilateral disease. Loss of corneal sensation strongly correlated with subbasal nerve plexus alterations as shown by IVCM.

Herpes zoster virus infection, or shingles, results from reactivation of the varicella zoster virus (VZV) from its latent state and can occur in patients with a history of varicella (chickenpox). Occurring in approximately 1 million people in the United States annually, shingles typically manifests as a painful skin rash. ${ }^{1}$ The trigeminal ganglion is the most frequent site of latency $(65 \%-90 \%)$ for VZV, with the first ophthalmic division being affected approximately 20 times more often than the other divisions. ${ }^{2}$ Although up to $30 \%$ of the population is affected by an episode of herpes zoster virus infection during their lifetime, a population-based study demonstrated that between $10 \%$ and $20 \%$ of cases involve the eye or adnexae (herpes zoster ophthalmicus [HZO]). ${ }^{3}$ Nearly two-thirds of patients with $\mathrm{HZO}$ demonstrate a keratitis that often is associated with loss of corneal sensation resulting from corneal denervation, necrotic ganglionitis, or damage to the mesencephalic nucleus in the brainstem. ${ }^{4}$ The resulting impaired corneal nerve function leads to neurotrophic keratopathy (NTK), which may manifest as dry eyes, persistent corneal epithelial defects, inflammation, corneal melting, and potentially perforation, possibly leading to significant vision loss or blindness. The management of NTK is extremely challenging because of the lack of monitoring tools and definitive treatments for advanced stages.

Corneal nerves, forming the subbasal nerve plexus, not only protect the ocular surface through sensory regulation of the blink reflex, but also release various trophic factors that regulate the modulation of epithelial integrity, proliferation, and wound healing. ${ }^{5}$ Although their complex stromal and epithelial branching is not visible by conventional slit-lamp biomicroscopy, it can be visualized by in vivo confocal microscopy (IVCM). In vivo confocal microscopy has become an increasingly popular clinical tool for imaging the living cornea at the cellular or microstructural level. This technique enables noninvasive optical sectioning with very high resolution and has been used in normal, diseased, and postsurgical eyes, increasing our understanding of corneal nerve anatomic features. The correlation between corneal innervation and corneal sensation recently was studied in normal human corneas, as well as in patients with dry eye syndrome, with keratoconus, with herpes simplex keratitis (HSK), ${ }^{6}$ and with diabetes, and in those who have undergone corneal surgery (reviewed in Cruzat et $\mathrm{al}^{7}$ and Pritchard et $\mathrm{al}^{8}$ ). Furthermore, in diabetes-induced peripheral neuropathy, IVCM recently was shown to diagnose and assess the disease progression. In some studies and clinical trials, the contralateral eyes have served as controls. ${ }^{9}$

Despite the high prevalence of $\mathrm{HZO}$ and its serious sequelae resulting from NTK, to date no systematic studies evaluating corneal nerve damage and corneal sensation have been performed in these patients. This study examines the extent of corneal nerve damage in patients with HZO by IVCM. Herein, significant corneal nerve diminishment is demonstrated, not only in the clinically affected eyes in patients with unilateral HZO, but also in their unaffected contralateral eyes.

\section{Patients and Methods}

\section{Patients}

This was a prospective, cross-sectional, single-center study conducted in a controlled, single-blinded fashion. Twenty-seven patients with a diagnosis of $\mathrm{HZO}$ with corneal involvement were included in the study. None of the patients was immune compromised, and all eyes were inactive, with no signs of active keratitis. Both the affected and the contralateral clinically unaffected eyes were studied. Fifteen eyes of 15 normal volunteers constituted the control group. Only 1 eye was included to prevent statistical bias. Subjects were recruited from the Cornea Service of the Department of Ophthalmology of the 
Massachusetts Eye \& Ear Infirmary, Boston, Massachusetts, who had HZO at presentation between 2006 and 2008. Subjects with a history of infectious keratitis, ocular inflammatory disease, ocular trauma, ocular surgery, contact lens use, or diabetes were excluded. The protocol was approved by the Institutional Review Board/Ethics Committee from the authors' institution, complied with the Health Insurance Portability and Accountability Act, and was conducted in accordance with the provisions of the Declaration of Helsinki. All participants provided written informed consent.

A literature review was performed in Medline to confirm the novelty of bilateral loss of the subbasal corneal nerve plexus after clinically unilateral herpes zoster ophthalmicus. The following words were used: herpes zoster ophthalmicus and corneal nerves or corneal innervation; shingles and corneal nerves or corneal innervation; herpes zoster and cornea and nerves.

\section{Corneal Sensation}

Examinations were carried out on all patients (D.P-L.) and normal subjects (P.H.) by slitlamp biomicroscopy, and bilateral central corneal sensation was measured with a CochetBonnet esthesiometer (Luneau Ophthalmologie, Chartres, France). This test mechanically stimulates corneal nerves by touching the tip of a retractable 6-cm long monofilament nylon thread of $0.12-\mathrm{mm}$ diameter against the corneal surface, decreasing in steps of $1.0 \mathrm{~cm}$ if a positive response was not obtained or advancing by $0.5 \mathrm{~cm}$ if a positive response was obtained. Measurements were repeated twice and were averaged, and the longest filament length resulting in a positive response was recorded. Patients were grouped according to the corneal sensitivity threshold measurements of the affected eye into normal $(>5.5 \mathrm{~cm})$, mild $(>2.5-5.5 \mathrm{~cm})$, and severe $(\mathcal{S} .5 \mathrm{~cm})$ loss of corneal sensation, and results were compared with those of contralateral eyes and normal controls.

\section{In Vivo Confocal Microscopy}

In vivo slit-scanning confocal microscopy (Confoscan 4; Nidek Technologies, Gamagori, Japan) was performed bilaterally on the central cornea of all subjects. The microscope is equipped with a $\times 40 / 0.75$ objective lens. One drop of topical anesthesia of $0.5 \%$ proparacaine hydrochloride (Alcaine; Alcon, Fort Worth, TX) was instilled in both eyes. A drop of $0.3 \%$ hypromellose (GenTeal gel; Novartis, East Hanover, NJ) was placed on the tip of the objective lens as an optical coupling medium, and the lens was advanced manually until the gel contacted the central surface of the cornea. A full-thickness confocal scan was acquired at a speed of 25 frames per second, obtaining 350 images per scan every $7 \mu \mathrm{m}$. A second scan was obtained for the anterior cornea, obtaining sections every $3 \mu \mathrm{m}$. Each image represents a coronal section of $460 \times 345 \mu \mathrm{m}\left(158700 \mu \mathrm{m}^{2}\right)$, with a minimum axial step of $1 \mu \mathrm{m}$, magnification of $\times 500$, and lateral resolution of $1 \mu \mathrm{m} /$ pixel. A total of 4 to 8 scans was obtained for each cornea.

Three representative images of the subbasal nerve plexus were selected for analysis from each eye. The images were selected from the layer immediately at or posterior to the basal epithelial layer and anterior to the Bowman's layer. The criteria to select the images were the best-focused and complete images, with the entire image in the same layer and with good contrast. Two masked observers (P.H. and M.D.) evaluated the confocal images and analyzed the subbasal nerve plexus.

The nerve analysis was carried out using the semiautomated tracing program NeuronJ, ${ }^{10} \mathrm{a}$ plug-in for ImageJ, a free image analysis software distributed by the National Institutes of Health (http://rsb.info.nih.gov/ij/http://rsb.info.nih.gov/ij/; http://www.imagescience.org/ meijering/software/neuronj/; accessed July 12, 2012). The entire frame was analyzed. Nerve 
density was assessed by measuring the total length of the nerve fibers in micrometers per frame. Main nerve trunks were defined as the total number of main nerve trunks in 1 image after analyzing the images anterior and posterior to the analyzed image to confirm that these did not branch from other nerves. Nerve branching was defined as the total number of nerve branches in 1 image. The number of total nerves measured was defined as the number of all nerves, including main nerve trunks and branches in 1 image. The grade of nerve tortuosity was classified in 4 grades according to a tortuosity grading scale reported by Oliveira-Soto and Efron. ${ }^{11}$

\section{Statistical Analysis}

Results were analyzed with Student $t$ test, the analysis of variance, Pearson's correlation coefficient, and multivariate linear regression analysis. The Tukey-Kramer method was used for adjustment for multiple comparisons. Differences were considered statistically significant for $P$ values less than 0.05 . Analyses were performed with SAS software version 9.2 (SAS Inc, Cary, NC).

\section{Results}

Twenty-seven eyes of $27 \mathrm{HZO}$ patients with corneal involvement were studied, as well as their respective contralateral clinically unaffected eyes. Results were compared with those of 15 normal eyes of 15 volunteers. Demographic data of patients and controls are presented in Table 1.

\section{Nerve Alterations by In Vivo Confocal Microscopy}

A summary of morphologic nerve parameters and corneal sensation for eyes with $\mathrm{HZO}$, their contralateral eyes, and normal control groups is reported in Table 2. A significant diminishment in the subbasal corneal nerve plexus was found in affected eyes with $\mathrm{HZO}$ as compared with controls, including decreased total nerve length (595.8 $\pm 358.1 \mathrm{vs.}$ $2258.4 \pm 989.0 \mu \mathrm{m} /$ frame; $P<0.0001)$, total nerve number (5.4 \pm 2.8 vs. $13.1 \pm 3.8 ; P<0.0001)$, main nerve trunks $(2.3 \pm 1.1$ vs. $4.7 \pm 1.2 ; P<0.0001)$, and number of branches $(3.2 \pm 2.3$ vs. $8.4 \pm 3.7 ; P<0.0001 ;$ Fig 1$)$. Nerve tortuosity was increased significantly in HZO compared with controls ( $2.3 \pm 0.7$ vs. $1.6 \pm 0.3 ; P<0.0005)$. Surprisingly, in the contralateral clinically unaffected eyes, the corneal subbasal nerve plexus also demonstrated a significant diminishment compared with controls, including total nerve length $(1053.1 \pm 441.4 \mu \mathrm{m} /$ frame; $P<0.0001)$, total nerve number $(8.3 \pm 2.9 ; P<0.0001)$, number of main nerve trunks (3.1 $\pm 1.0 ; P<0.0001)$, and number of nerve branches $(5.2 \pm 2.2 ; P<0.003)$. These results were surprising in that they demonstrate unilateral ophthalmic shingles causing not only a significant reduction of the corneal innervation in the affected eyes, but also in the contralateral clinically unaffected eyes (Fig 1).

\section{Correlation of Corneal Sensitivity to In Vivo Confocal Microscopy Findings}

To correlate corneal nerve function, measured by sensation, with corneal nerve alterations seen on IVCM, patients were subdivided into 3 groups based on corneal sensation in the HZO-affected eyes. Patients were classified as having normal, mild, or severe sensation loss. Regardless of the subgroup of patients, a significant reduction of the corneal subbasal nerve plexus was found in all HZO eyes (normal, mild, and severe sensation loss); specifically, total nerve length $(P<0.005, P<0.0001$, and $P<0.0001$, respectively; Fig $2 \mathrm{~A})$, total nerve number $(P<0.0005, P<0.0001$, and $P<0.0001$, respectively; Fig $2 \mathrm{~B})$, number of main nerve trunks ( $P<0.0001, P<0.0001$, and $P<0.0001$, respectively; Fig $2 \mathrm{C})$, and number of nerve branches $(P<0.03, P<0.0001$, and $P<0.0003$, respectively; Fig $2 \mathrm{D})$ were reduced significantly compared with the normal control group (Fig 2 ). The groups with mild and severe sensation loss demonstrated higher significance for total nerve length and total nerve 
numbers. In the contralateral unaffected eyes of HZO patients, all groups demonstrated loss of corneal nerves as well. However, the decrease in corneal nerves was statistically significant only in the mild and severe sensation loss groups $(P<0.05$ for all parameters; Fig 2).

\section{Correlation and Regression Analysis}

Pearson's correlation coefficient was used to correlate nerve morphologic features and their functions, specifically, the reduction of subbasal corneal nerves observed by IVCM and the loss of corneal sensation measured by esthesiometry. A significant correlation was observed between the reduction in the subbasal nerve plexus and the decrease in corneal sensation. Corneal sensation was correlated significantly with total nerve length $(R=0.48 ; P<0.0001)$, total number of nerves $(R=0.43 ; P<0.0004)$, number of main nerve trunks $(R=0.38$; $P<0.002)$, and number of nerve branches $(R=0.39 ; P<0.002)$, whereas it was inversely correlated to nerve tortuosity $(R=-0.32 ; P<0.02)$. To calculate the approximate corneal nerve density needed for normal sensation, quantitative regression analysis was applied. Abnormal sensation ( $5.5 \mathrm{~cm}$ ) was noted with a total nerve length of $1032.6 \mu \mathrm{m} / \mathrm{frame}$ or lower (Fig 3), which is much lower than the average nerve density of the normal cornea. No significant correlations were found between the IVCM findings of subbasal nerve plexus with age, number of reactivations, and time from first episode (all $P>0.05$ ).

\section{Discussion}

Until recently, the clinical evaluation of nerves in herpes zoster has only been possible through measurement of corneal sensation or by means of invasive skin biopsies. More recently, IVCM has allowed noninvasive, high-resolution, and precise systematic clinical studies of corneal innervation in clinical practice. The single previous study describing pathologic corneal nerve alterations in a single HZO patient by IVCM is a case report by Patel and McGhee. ${ }^{12}$ To the authors' knowledge, this is the first systematic IVCM study in HZO patients demonstrating accurate noninvasive assessment of corneal nerve alterations in this disease. Our results show significant correlation of corneal sensation with the corneal nerve damage. More importantly, the study demonstrated significant bilateral corneal nerve alterations in clinically unilateral $\mathrm{HZO}$.

The contralateral loss of corneal nerves, although not as profound as in HZO eyes, was a completely unexpected finding. Although there are no well-established anatomic connections between neurons that innervate homologous right and left body parts, bilateral abnormalities after unilateral injury have been reported previously. ${ }^{13}$ Furthermore, the authors recently reported bilateral changes in corneal nerves after HSK, ${ }^{6}$ although HSK can be bilateral in $10 \%$ to $20 \%$ of cases. Moreover, Oaklander et al ${ }^{14}$ previously reported that unilateral shingles can cause contralateral damage to epidermal nerves in skin biopsies, although less severe than in the affected side. In addition, studies in patients with shingles have demonstrated bilateral decrease in axon-reflex vasodilatation both ipsilateral and contralateral to the lesion. ${ }^{15}$

The contralateral diminishment of corneal nerves could be mediated by the central nervous system (CNS) in a neurogenic fashion. These contralateral mirror-image changes after unilateral nerve damage may be present without infection or virus material. ${ }^{14,15}$ Bilateral changes after unilateral nerve damage have been demonstrated in several pain models showing contralateral pain-related behaviors, ${ }^{16}$ anatomic changes in sensory ganglia, ${ }^{17}$ and disturbed function of cutaneous nociceptive terminals. ${ }^{18}$ These rapid contralateral effects in the mirror area after 1-sided nerve injuries suggest the involvement of spinal cord or brain circuits, ${ }^{19}$ with primary afferent inputs from peripheral nerves projecting bilaterally, as 
axons have been described crossing between bilateral dorsal horns via the dorsal commissure of the spinal cord. ${ }^{20}$

In the case of the cornea, it is the current clinical dogma that sensory innervation is mediated along a unilateral ophthalmic nerve pathway. ${ }^{21}$ However, there is evidence that peripheral trigeminal nerve fibers directly project to bilateral areas of brainstem nuclei and the caudal medulla. ${ }^{22,23}$ In addition, frequent contralateral projections between the trigeminal nuclei of both sides have been detected in studies using anterograde tracers. ${ }^{23}$ Thus, primary trigeminal fibers could cross the pontine tegmentum to reach the contralateral principal nucleus. ${ }^{24}$ The presence of these anatomic CNS circuits makes it likely that unilateral nerve damage may result in bilateral changes in CNS signaling. Support for this concept also comes from animal models of experimental inflammatory peripheral pain, in which peripheral nerve pain led to bilateral changes of the blood-brain barrier. ${ }^{25}$ These findings implicate that unilateral nerve damage results in bilateral (or midline) CNS neuronal changes, which in turn may cause functional and morphologic disturbances in the contralateral mirror area.

The bilateral nerve alterations in the cases of unilateral HZO described herein also may be explained by CNS-mediated contralateral effects, although we cannot rule out subclinical infection in the contralateral eyes. However, the latter option is rather unlikely, because the current findings were consistent in all $\mathrm{HZO}$ patients. Varicella zoster virus-related brainstem alterations, although rare, in theory may lead to bilateral trigeminal nerve damage. Virus particles have been found in acute VZV in the trigeminal ganglion and its axons. ${ }^{26}$ Watson et $\mathrm{al}^{27}$ demonstrated bilateral abnormalities in sensory roots (loss of large myelinated axons) after unilateral shingles. Further, magnetic resonance imaging scans obtained during unilateral zoster infection have shown bilateral gadolinium-diethylene triamine pentaacetic acid (DTPA) enhancement of spinal roots. ${ }^{28}$ Together, these reports suggest that retrograde spread of VZV to the brainstem and spinal cord via the trigeminal ganglion could damage the corresponding mesencephalic sensory nucleus, subsequently leading to corneal denervation, although because of the rarity of these cases, it is rather unlikely in the current patients. $^{26}$

Although the assessment of corneal innervation until recently has been possible only through measurement of corneal sensation, IVCM now allows us an objective method of studying corneal nerves. The correlation of morphologic corneal nerve quantification with their function demonstrated a significant correlation between the reduction of corneal subbasal nerves and the loss of corneal sensation measured by esthesiometry. To date, the correlation of subbasal corneal nerves and their function has been studied in normal eyes, ${ }^{29}$ keratoconic eyes, ${ }^{30}$ eyes with dry eye, ${ }^{31}$ herpes simplex keratitis eyes, ${ }^{6}$ contact lens wearers, ${ }^{32}$ eyes of patients with diabetes mellitus, ${ }^{33}$ and eyes that have undergone refractive surgery. ${ }^{34}$ The current study is the first to correlate the quantitative findings of the corneal subbasal nerve plexus with corneal sensation in patients after HZO.

The fact that abnormal sensation was noted with a nerve density only of $1032 \mu \mathrm{m} /$ frame or lower in this study explains why the sensation in the contralateral unaffected eyes was perceived as normal by patients, despite significant neural diminishment. Thus, quantification of corneal innervation by IVCM demonstrates an objective and sensitive methodology for monitoring patients with HZO, potentially predicting the risk for NTK or demonstrating neural recovery. Since NTK initially often lacks signs and symptoms, preventing epithelial breakdown is crucial, because this can cause corneal perforation to progress. Although most of the current patients had begun receiving antiviral therapy, it is unclear how the initiation of antiviral therapy or the lack thereof would affect these findings. A limitation of the current study is the evaluation of only the center of the cornea for corneal 
sensitivity and IVCM. One cannot necessarily extrapolate these findings to the peripheral cornea. In addition, topographic reproducibility and the difficulty of ensuring the exact same locations tested with both techniques currently are not optimal. Furthermore, the CochetBonnet esthesiometer, although the most widely used technique for measuring corneal sensation, measures only mechanical nociceptors and has a limited scale. In conclusion, these results provide strong evidence for bilateral nerve alterations in unilateral shingles, strongly correlating with corneal sensation loss.

The consequences of remote changes in the contralateral unaffected innervation area most likely are not limited to the ocular field. In stroke patients, it is well described that focal ischemia to the brain can result in functional defects in structurally intact but neuroanatomically connected areas, termed diaschisis. ${ }^{35}$ The present study suggests that the concept of diaschisis may be similarly relevant to the peripheral nervous system with broad implications in current practice. For example, most disease scales for neurologic disorders include left-versus-right comparison (such as reflexes and muscle strength in multiple sclerosis or disc prolapse) to judge the effects of treatment on one side or improvement during therapy. Similarly, the contralateral eyes either are used as controls in clinical practice, for research studies, or clinical trials, or are used as so-called independent eyes to measure outcome parameters. Thus, these findings suggest that this current practice should be rethought, because the comparison between affected and unaffected eyes or body sites for determination of sensation, pain, muscle strength, and reflexes may lead to erroneous results. Thus, it is tempting to speculate whether bilateral electrophysiologic abnormalities in routine nerve latency and velocity testing in some cases may result from only unilateral disease. Therefore, objective quantitative measurements are required. In the case of the cornea, sensation measurements with esthesiometers (e.g., by the Cochet-Bonnet esthesiometer or the Belmonte esthesiometer ${ }^{36}$ or for corneal innervation through IVCM are advisable. Further studies of the bilateral involvement of shingles will help to further the understanding of the pathophysiologic features of this disease.

\section{Acknowledgments}

Support by the National Institutes of Health, Bethesda, Maryland (grant nos.: K08-EY020575 [PH], K12EY016335 [P.H.], and K24-EY019098 [R.D.]; a Career Development Award by Research to Prevent Blindness, New York, New York (P.H.); the New England Corneal Transplant Research Fund (P.H.); Dr. Ralph and Marian Falk Medical Research Trust, Chicago, IL (P.H.); the Johnstone Research Fund, Boston, Massachusetts (D.P-L.); and the Stevens Research Fund, Boston, Massachusetts (D.P-L.). The funding organizations had no role in the design or conduct of this research.

\section{References}

1. Schmader KE, Dworkin RH. Natural history and treatment of herpes zoster. J Pain. 2008; 9(suppl):S3-9. [PubMed: 18166460]

2. Liesegang TJ. Varicella-zoster virus eye disease. Cornea. 1999; 18:511-31. [PubMed: 10487424]

3. Liesegang TJ. Herpes zoster ophthalmicus: natural history, risk factors, clinical presentation, and morbidity. Ophthalmology. 2008; 115(suppl):S3-12. [PubMed: 18243930]

4. Pavan-Langston D. Herpes zoster ophthalmicus. Neurology. 1995; 45(suppl):S50-1. [PubMed: 8545020]

5. Muller LJ, Marfurt CF, Kruse F, Tervo TM. Corneal nerves: structure, contents and function. Exp Eye Res. 2003; 76:521-42. [PubMed: 12697417]

6. Hamrah P, Cruzat A, Dastjerdi MH, et al. Corneal sensation and subbasal nerve alterations in patients with herpes simplex keratitis: an in vivo confocal microscopy study. Ophthalmology. 2010; 117:1930-6. [PubMed: 20810171]

7. Cruzat A, Pavan-Langston D, Hamrah P. In vivo confocal microscopy of corneal nerves: analysis and clinical correlation. Semin Ophthalmol. 2010; 25:171-7. [PubMed: 21090996] 
8. Pritchard N, Edwards K, Shahidi AM, et al. Corneal markers of diabetic neuropathy. Ocul Surf. 2011; 9:17-28. [PubMed: 21338566]

9. Rosenberg ME, Tervo TM, Muller LJ, et al. In vivo confocal microscopy after herpes keratitis. Cornea. 2002; 21:265-9. [PubMed: 11917174]

10. Meijering E, Jacob M, Sarria JC, et al. Design and validation of a tool for neurite tracing and analysis in fluorescence microscopy images. Cytometry A. 2004; 58:167-76. [PubMed: 15057970]

11. Oliveira-Soto L, Efron N. Morphology of corneal nerves using confocal microscopy. Cornea. 2001; 20:374-84. [PubMed: 11333324]

12. Patel DV, McGhee CN. Laser scanning in vivo confocal microscopy demonstrating significant alteration of human corneal nerves following herpes zoster ophthalmicus. Arch Neurol. 2010; 67:640-1. [PubMed: 20457969]

13. Oaklander AL, Brown JM. Unilateral nerve injury produces bilateral loss of distal innervation. Ann Neurol. 2004; 55:639-44. [PubMed: 15122703]

14. Oaklander AL, Romans K, Horasek S, et al. Unilateral post-herpetic neuralgia is associated with bilateral sensory neuron damage. Ann Neurol. 1998; 44:789-95. [PubMed: 9818935]

15. Baron R, Saguer M. Axon-reflex reactions in affected and homologous contralateral skin after unilateral peripheral injury of thoracic segmental nerves in humans. Neurosci Lett. 1994; 165:97100. [PubMed: 8015746]

16. Attal N, Filliatreau G, Perrot S, et al. Behavioural pain-related disorders and contribution of the saphenous nerve in crush and chronic constriction injury of the rat sciatic nerve. Pain. 1994; 59:301-12. [PubMed: 7892028]

17. McLachlan EM, Janig W, Devor M, Michaelis M. Peripheral nerve injury triggers noradrenergic sprouting within dorsal root ganglia. Nature. 1993; 363:543-6. [PubMed: 8505981]

18. Allnatt JP, Dickson KE, Lisney SJ. Saphenous nerve injury and regeneration on one side of a rat suppresses the ability of the contralateral nerve to evoke plasma extravasation. Neurosci Lett. 1990; 118:219-22. [PubMed: 2274274]

19. Willis, WD.; Coggeshall, RE. Sensory Mechanisms of the Spinal Cord. 2. New York: Plenum Press; 1991. p. 575

20. Ramon y Cajal, S. Histology of the Nervous System of Man and Vertebrates. New York: Oxford University Press; 1995. p. 1672

21. Walker, HK. Cranial nerve V: the trigeminal nerve. In: Walker, HK.; Hall, WD.; Hurst, JW., editors. Clinical Methods: The History, Physical, and Laboratory Examinations. 3. Boston, MA: Butterworths; 1990. p. 318-21.

22. Jacquin MF, Chiaia NL, Rhoades RW. Trigeminal projections to contralateral dorsal horn: central extent, peripheral origins, and plasticity. Somatosens Mot Res. 1990; 7:153-83. [PubMed: 2378191]

23. Pfaller K, Arvidsson J. Central distribution of trigeminal and upper cervical primary afferents in the rat studied by anterograde transport of horseradish peroxidase conjugated to wheat germ agglutinin. J Comp Neurol. 1988; 268:91-108. [PubMed: 3346387]

24. Clarke WB, Bowsher D. Terminal distribution of primary afferent trigeminal fibers in the rat. Exp Neurol. 1962; 6:372-83. [PubMed: 14021584]

25. Campos CR, Ocheltree SM, Hom S, et al. Nociceptive inhibition prevents inflammatory pain induced changes in the blood-brain barrier. Brain Res. 2008; 1221:6-13. [PubMed: 18554577]

26. Nagashima K, Nakazawa M, Endo H. Pathology of the human spinal ganglia in varicella-zoster virus infection. Acta Neuropathol. 1975; 33:105-17. [PubMed: 173126]

27. Watson C, Deck J, Morshead C, et al. Post-herpetic neuralgia: further post-mortem studies of cases with and without pain. Pain. 1991; 44:105-17. [PubMed: 1711192]

28. Hanakawa T, Hashimoto S, Kawamura J, et al. Magnetic resonance imaging in a patient with segmental zoster paresis. Neurology. 1997; 49:631-2. [PubMed: 9270616]

29. Patel DV, Tavakoli M, Craig JP, et al. Corneal sensitivity and slit scanning in vivo confocal microscopy of the subbasal nerve plexus of the normal central and peripheral human cornea. Cornea. 2009; 28:735-40. [PubMed: 19574916] 
30. Patel DV, Ku JY, Johnson R, McGhee CN. Laser scanning in vivo confocal microscopy and quantitative aesthesiometry reveal decreased corneal innervation and sensation in keratoconus. Eye (Lond). 2009; 23:586-92. [PubMed: 18344958]

31. Benitez-Del-Castillo JM, Acosta MC, Wassfi MA, et al. Relation between corneal innervation with confocal microscopy and corneal sensitivity with noncontact esthesiometry in patients with dry eye. Invest Ophthalmol Vis Sci. 2007; 48:173-81. [PubMed: 17197530]

32. Patel SV, McLaren JW, Hodge DO, Bourne WM. Confocal microscopy in vivo in corneas of longterm contact lens wearers. Invest Ophthalmol Vis Sci. 2002; 43:995-1003. [PubMed: 11923239]

33. Rosenberg ME, Tervo TM, Immonen IJ, et al. Corneal structure and sensitivity in type 1 diabetes mellitus. Invest Ophthalmol Vis Sci. 2000; 41:2915-21. [PubMed: 10967045]

34. Stachs O, Zhivov A, Kraak R, et al. Structural-functional correlations of corneal innervation after LASIK and penetrating keratoplasty. J Refract Surg. 2010; 26:159-67. [PubMed: 20229947]

35. Witte OW, Bidmon HJ, Schiene K, et al. Functional differentiation of multiple perilesional zones after focal cerebral ischemia. J Cereb Blood Flow Metabolism. 2000; 20:1149-65.

36. Belmonte C, Acosta MC, Schmelz M, Gallar J. Measurement of corneal sensitivity to mechanical and chemical stimulation with a $\mathrm{CO}_{2}$ esthesiometer. Invest Ophthalmol Vis Sci. 1999; 40:513-9. [PubMed: 9950612] 


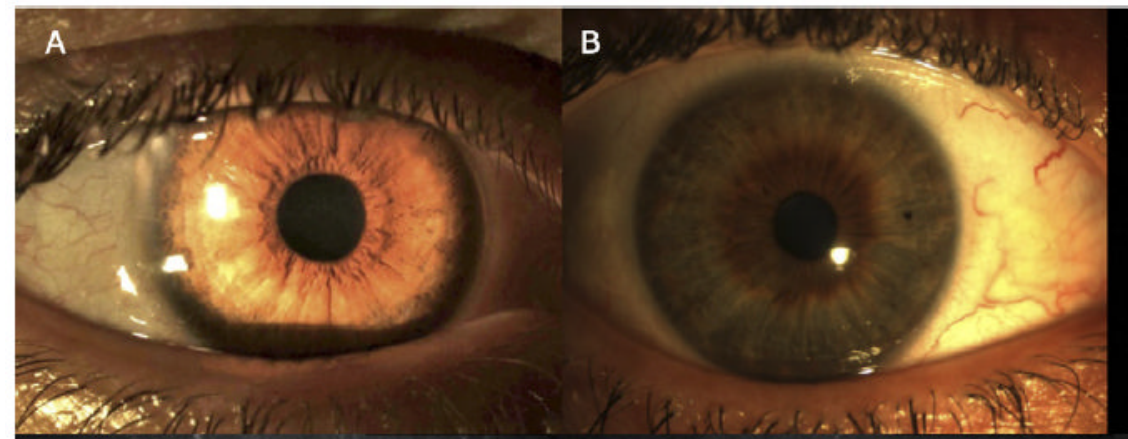

E

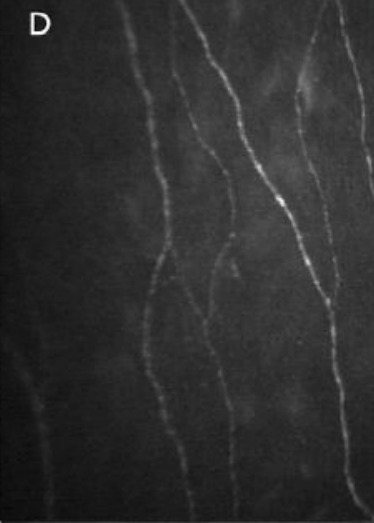

G

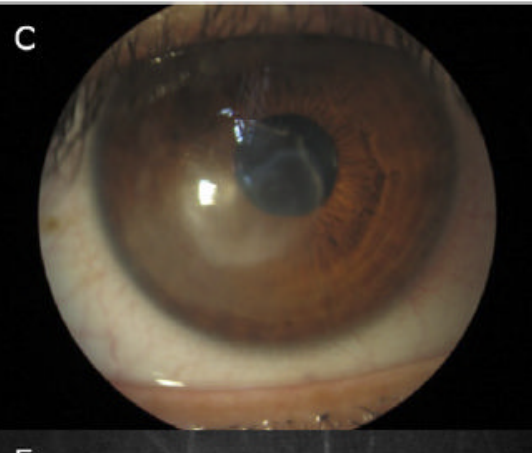

F

I

Figure 1.

Slit-lamp photographs and in vivo confocal microscopy images obtained at the level of the corneal subbasal nerve plexus. A, Slit-lamp photograph of normal cornea. B, Slit-lamp photograph of contralateral clinically unaffected eye of herpes zoster ophthalmicus (HZO) patient. C, Slit-lamp photograph of eye affected by HZO. D, In vivo confocal microscopy image showing normal corneal subbasal nerve plexus. E, In vivo confocal microscopy image showing contralateral clinically unaffected eye of HZO patient. Note the decrease in length and number of nerves. F, In vivo confocal microscopy image showing $\mathrm{HZO}$ with normal corneal sensation. Note the decrease in nerve length. $\mathbf{G}$, In vivo confocal microscopy image showing HZO mild sensation loss. Note the decrease in length and number of nerves and branches. H, I, In vivo confocal microscopy image showing HZO severe sensation loss. 
A) Total Nerve Length

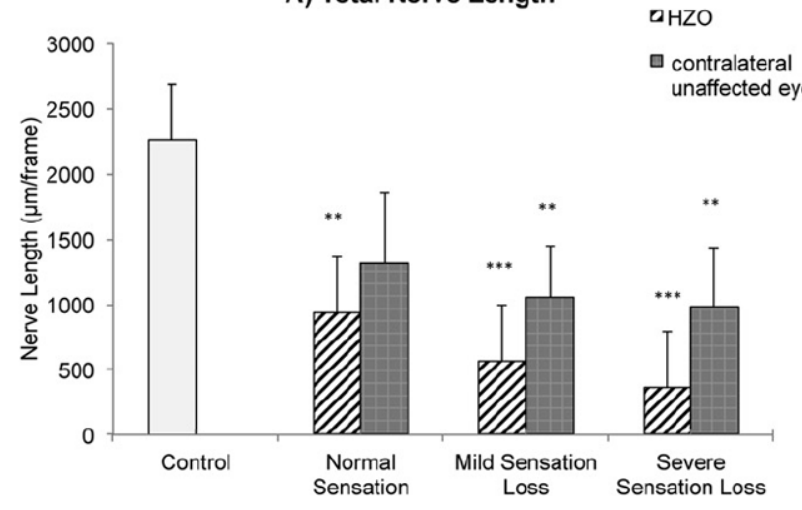

C) Main Nerve Trunks

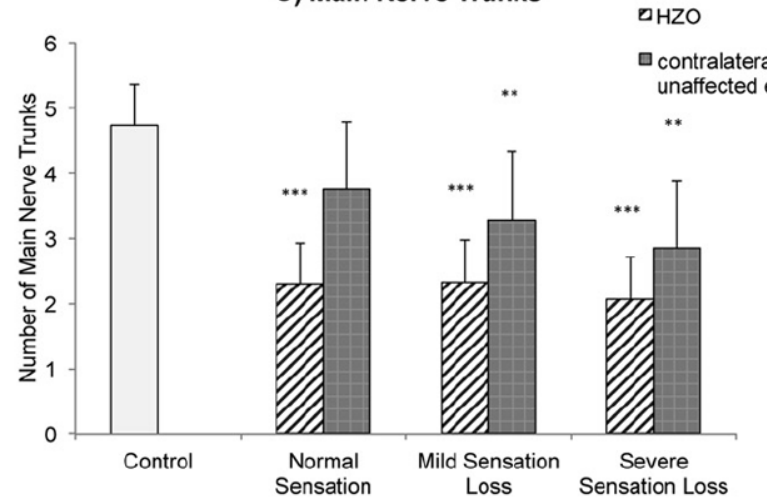

B) Total Number of Nerves

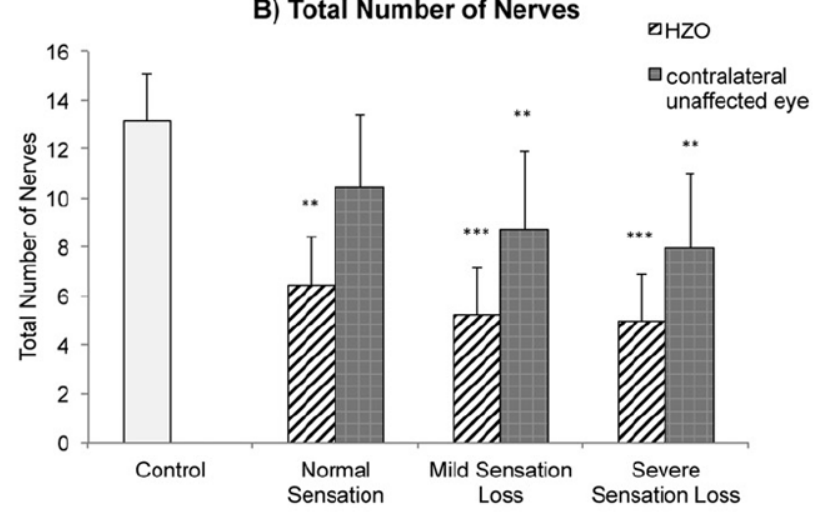

D) Nerve Branches

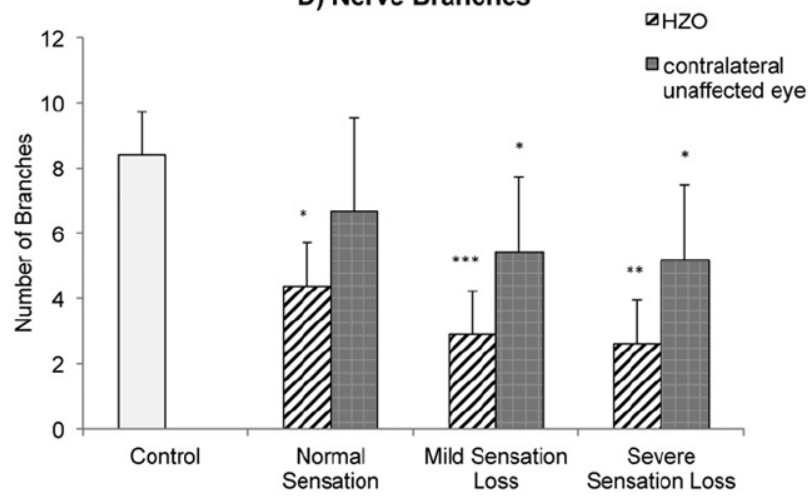

Figure 2.

Bar graphs showing subbasal corneal nerve alterations in affected and clinically unaffected eyes in herpes zoster ophthalmicus (HZO) patients and according to grade of hypoesthesia and in the control group: (A) total nerve length, (B) total number of nerves, $(\mathbf{C})$ main nerve trunks, and (D) nerve branches. Error bars represents standard deviation from the mean. $* P<0.05, * * P<0.001$, and $* * * P<0.0001$ compared with control group by analysis of variance. 
Corneal Sensation vs. Nerve Length

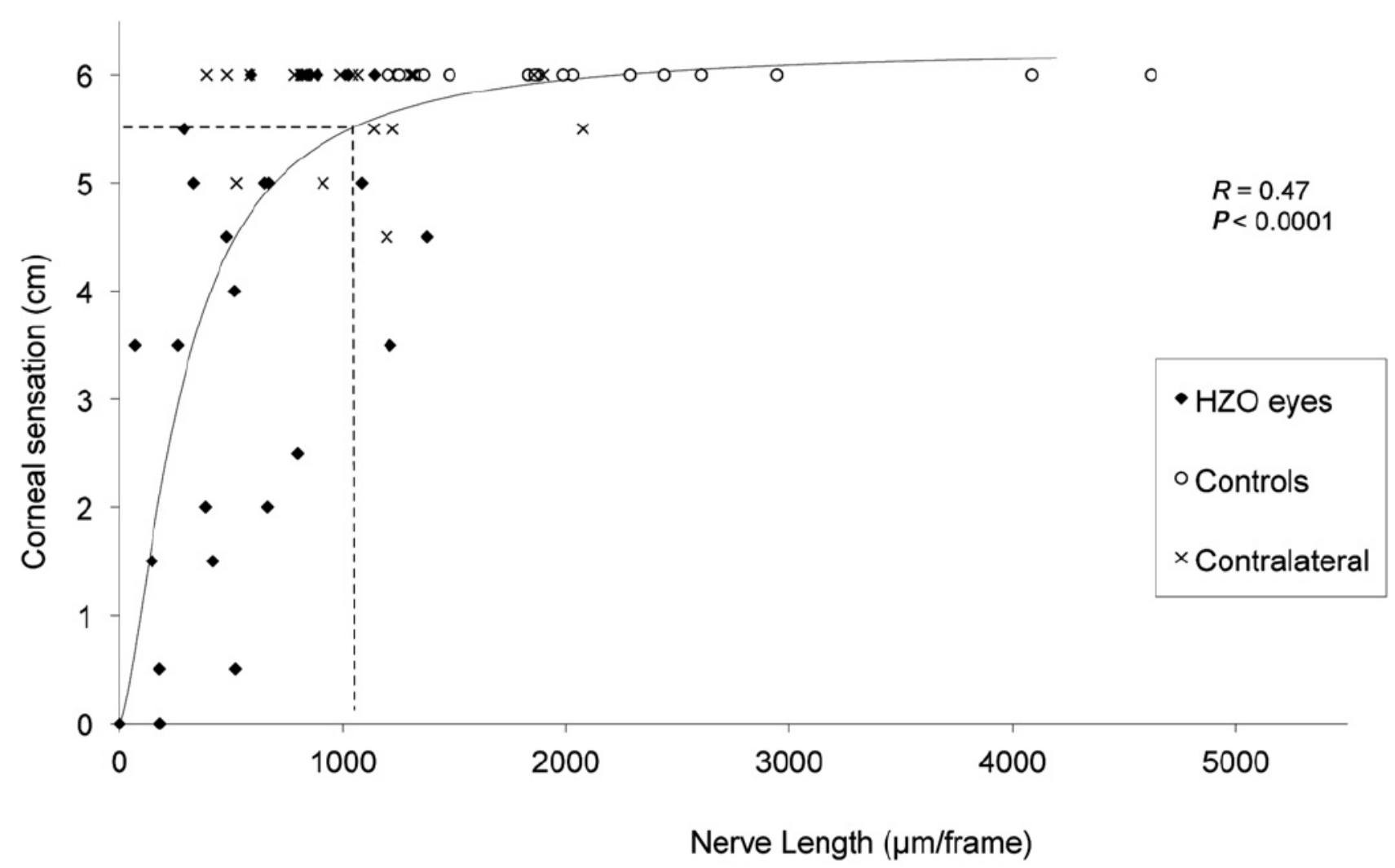

Figure 3.

Graph showing that corneal sensation is correlated significantly to nerve length in herpes zoster ophthalmicus (HZO). Pearson's correlation coefficient $(R)$ and $P$ value are shown. Dashed lines show the cutoff point calculated through quantitative regression of corneal sensation from nerve density. Abnormal sensation $(\$ 5.5 \mathrm{~cm})$ is noted with a nerve density of $1032 \mu \mathrm{m} /$ frame or less. 


\section{Table 1}

Demographic Data of Normal Controls and Patients with Herpes Zoster Ophthalmicus

\begin{tabular}{lcc}
\hline & Controls & Herpes Zoster Ophthalmicus \\
\hline No. of patients & 15 & 27 \\
Age (yrs) & $59.1 \pm 17.2$ & $60.9 \pm 15.6$ \\
Gender (male/female) & $8 / 7$ & $9 / 18$ \\
Sensation (cm) & $6.0 \pm 0$ & $3.8 \pm 2.1$ \\
Time since onset (yrs) & - & $5.1 \pm 6.4$ \\
No. of episodes & - & $1.9 \pm 0.9$ \\
\hline
\end{tabular}

Values are reported as mean \pm standard deviation unless otherwise indicated.

Episodes reflect both primary and secondary immune keratitis. 


\section{Table 2}

Corneal Subbasal Nerve Plexus Parameters and Corneal Sensation in Eyes with Herpes Zoster Ophthalmicus, Contralateral Clinically Unaffected Eyes, and Control Group

\begin{tabular}{|c|c|c|c|}
\hline & \multicolumn{2}{|c|}{ Herpes Zoster Ophthalmicus Patients } & \multirow[b]{2}{*}{ Controls } \\
\hline & Herpes Zoster Ophthalmicus Eye & Contralateral Unaffected Eye & \\
\hline No. of eyes & 27 & 27 & 15 \\
\hline $\begin{array}{l}\text { Mean central corneal sensation } \\
(\mathrm{cm})\end{array}$ & $3.8 \pm 2.1^{*}$ & $5.8 \pm 0.4^{\dagger}$ & $6.0 \pm 0$ \\
\hline $\begin{array}{l}\text { Total nerve length, } \mu \mathrm{m} / \text { frame } \\
\left(\mu \mathrm{m} / \mathrm{mm}^{2}\right)\end{array}$ & $595.8 \pm 358.1 *(4462.9 \pm 2682.4)$ & $1053.1 \pm 441.4^{* \dagger}(7888.4 \pm 3306.4)$ & $2258.4 \pm 989.0(16916.9 \pm 7408.2)$ \\
\hline Total no. of nerves & $5.4 \pm 2.8^{*}$ & $8.3 \pm 2.9^{*} \dagger$ & $13.1 \pm 3.8$ \\
\hline No. of main nerve trunks & $2.3 \pm 1.1^{*}$ & $3.1 \pm 1.0 * \dagger$ & $4.7 \pm 1.2$ \\
\hline Total no. of nerve branches & $3.2 \pm 2.3^{*}$ & $5.2 \pm 2.2^{\dagger t}$ & $8.4 \pm 3.7$ \\
\hline Grade of tortuosity & $2.3 \pm 0.7$ & $1.8 \pm 0.4^{\dagger}$ & $1.6 \pm 0.3$ \\
\hline
\end{tabular}

Values reported as mean \pm standard deviation unless otherwise indicated.

* Statistically significant compared with normals, $P<0.0001$.

${ }^{\dagger}$ Statistically significant compared with herpes zoster ophthalmicus group, $P<0.05$.

${ }^{*}$ Statistically significant compared with normals, $P<0.001$. 